\title{
Comparison of Peripheral Nerve Block and Spinal Anesthesia in Terms of Postoperative Mortality and Walking Ability in Elderly Hip Fracture Patients - A Retrospective, Propensity-Score Matched Study
}

\author{
Guangtao $\mathrm{Fu} \mathbb{D}^{1, *}$ \\ Haotao Li (iD) ${ }^{1,2, *}$ \\ Hao Wang' \\ Ruiying Zhang' \\ Mengyuan $\mathrm{Li}^{1}$ \\ Junxing Liao' \\ Yuanchen $\mathrm{Ma}^{\mathrm{I}}$ \\ Qiujian Zheng' \\ Qingtian $\mathrm{Li}^{\text {I }}$ \\ 'Department of Orthopedics, \\ Guangdong Provincial People's Hospital, \\ Guangdong Academy of Medical Sciences, \\ Guangzhou, Guangdong Province, \\ People's Republic of China; ${ }^{2}$ College of \\ Clinical Medicine, Shantou University, \\ Shantou, Guangdong Province, People's \\ Republic of China
}

*These authors contributed equally to this work

\begin{abstract}
Purpose: To compare the effects of peripheral nerve block (PNB) and spinal anesthesia (SA) on one-year mortality and walking ability of elderly hip fracture patients after hip arthroplasty. Methods: Patients $\geq 65$ years who underwent unilateral hip arthroplasty due to femoral neck fracture, using either PNB or SA from 2014 to 2019, were included. Demographic data, comorbidities, and results of preoperative screening were retrospectively collected. Propensity score matching (PSM) was performed in a ratio of 1:1 for PNB and SA groups. The primary outcomes were 30-day, 90-day, and one-year mortality. Secondary outcomes included walking ability in the first postoperative year, major complications, length of stay, and the cost of hospitalization. Survival analysis was performed using Kaplan-Meier method.

Results: Three hundred and sixteen patients were included, of whom 200 received SA and 116 received PNB. Eighty-nine patients in each group were matched after PSM. Patients in the PNB group showed significantly lower risks of death in 30 days $(2.2 \%$ vs $10.1 \%$, $\mathrm{P}=0.029)$ and 90 days $(3.4 \%$ vs $12.4 \%, \mathrm{P}=0.026)$ after hip arthroplasty, when compared to the SA group. There was no significant difference in one-year mortality, walking ability, major complications, and length of stay. Higher hospitalization cost was found in the PNB group (53,828.21 CNY vs 59,278.83 CNY, $\mathrm{P}=0.024)$. One-year accumulated survival rate was higher in the PNB group without reaching a significant level.

Conclusion: PNB was related to lower 30- and 90-day mortality but higher hospitalization cost in elderly hip fracture patients after hip arthroplasty. However, the anesthesia types were not associated with one-year mortality, one-year walking ability, major complications, and length of stay.
\end{abstract}

Keywords: elderly hip fracture, 30-day mortality, walking ability, peripheral nerve block, propensity score matching

\section{Introduction}

As the worldwide population is aging, geriatric hip fracture becomes an increasingly significant global public health problem. Hip fracture affects 4.5 million people per year worldwide, and the number is expected to increase to 21 million in the next 40 years. ${ }^{1}$ Accounting for a majority of hip fractures, geriatric femoral neck fracture is a common clinical scenario encountered by orthopedic surgeons.
Department of Orthopedics, Guangdong Provincial People's Hospital, Guangdong Academy of Medical Sciences, 106,

Zhongshan 2nd Road, Yuexiu District,

Guangzhou, Guangdong Province,

People's Republic of China

Tel +86-18820109789

Email398619503@qq.com 
Despite major advances in anesthesia and surgical procedure, patients who underwent urgent hip arthroplasty still have significantly higher risks of functional disability and mortality than patients who underwent other elective hip surgeries. ${ }^{2}$ Data from Australia in 2017 showed that 7\% of the patients died within 30 days of surgery. ${ }^{3}$ Detailed and evidence-based peri-operative management protocols are strongly needed for prognosis improvement in elderly patients with hip fractures.

Type of anesthesia has proven to be closely related to postoperative mortality in patients with hip fracture, ${ }^{4}$ and there is ongoing debate regarding the appropriate anesthesia technique. Regional anesthesia (ie spinal or epidural) and general anesthesia are presently the most widely used techniques. ${ }^{5}$ However, the applications of both types of anesthesia are severely limited in cases of severe comorbidities (eg severe respiratory and cardiac dysfunction) and anticoagulant administration, which are common in elderly hip fracture patients. ${ }^{6}$

Peripheral nerve blocks (PNBs) share many of their advantages with spinal and general anesthetic techniques. Moreover, the efficiency of PNB in enhanced recovery after surgery (ERAS) protocol for patients who have undergone elective hip arthroplasty has been well established. $^{7}$ It is believed that elderly patients with hip fractures could also benefit from PNB for not only its opioid-sparing effect and decreased risk of opioid-related adverse event but also lower risks of cognitive dysfunction, pneumonia, and hemodynamic instability. ${ }^{7}$ PNB is also available for elderly patients whose airway management is difficult and who have borderline respiratory function. ${ }^{8}$ However, whether these benefits would translate into better clinical outcomes (mortality and walking ability) and decreased resource use (length of stay (LOS) and cost of hospitalization) have yet to be well investigated.

Most of the studies concentrated on the use of PNB as preoperative analgesia, as postoperative analgesia, or as a supplement to general anesthesia for hip fracture surgery. ${ }^{8-10}$ To the best of our knowledge, there are only a few retrospective studies and case reports concerning the effect of sole application of PNB on postoperative mortality and functional outcomes. ${ }^{11-14}$ Quality and quantity of evidence in these retrospective studies were largely limited due to the inevitable selection bias and confounding factors. Propensity score matching (PSM) is one of the major statistical approaches to minimize selection bias. It was also reported that data after PSM were approximated to what a prospective randomized data set would have shown. ${ }^{15}$ Thus, the aim of the present study was to investigate the effect of PNB on postoperative mortality and walking ability in elderly hip fracture patients, and to compare with SA in a retrospective, PSM design.

\section{Methods and Materials Study Design}

The present study was conducted in accordance with the ethical principles of the Helsinki declaration and was approved by the institutional review board of Guangdong Provincial People's Hospital. Signed informed consents for participation were unavailable due to the retrospective design; accordingly, the institutional review board of Guangdong Provincial People's Hospital has waived the informed consent procedure for the present study. The electronic medical records of our hospital were reviewed to identify patients who met the following inclusion criteria: 1) aged $\geq 65$ years, 2) having undergone unilateral hip arthroplasty due to low-energy mechanism femoral neck fracture, in conjunction with either nerve block anesthesia or spinal anesthesia at our center between January 1st, 2014 and December 31st, 2019. The exclusion criteria included those with 1) a previous history of trauma or surgery in the involved hip, 2) a peri-prosthetic or open fracture, 3) severe multiple injuries that required emergency surgery for non-orthopedic-related reasons, 4) pathological fracture, and 5) absence of intact data.

\section{Data Collection}

Data of selected patients were retrospectively retrieved from the medical database of our hospital. Demographic features included patients' pre-fracture condition (place of residence, previous history of hip fracture in the contralateral side), time from injury to surgery, age, sex, marital status, medical insurance, and smoking history. Major comorbidities included type 2 diabetes, circulatory abnormalities (hypertension, coronary heart disease, prior myocardial infarction, and arrhythmia), chronic obstructive pulmonary disease, pulmonary infection, prior stroke, dementia, Parkinson's disease, digestive system disorders, chronic renal failure, rheumatologic disease, and osteoporosis. Charlson comorbidity index (CCI) was calculated to obtain an overall assessment of preoperative comorbid condition. ${ }^{16}$ Preoperative vital signs, results of the electrocardiogram and chest radiograph, as well as blood counts and biochemical analyses (including hemoglobin, serum albumin (ALB), blood glucose, and international 
normalized ratio (INR)) obtained in the emergency department were recorded. An abnormal vital sign was defined according to the criteria of Zanker's study. ${ }^{17}$ Results of electrocardiogram and chest radiograph were classified as "Abnormality" only when they were considered to be clinically significant by the correspondent authors. Treatment details including surgical procedures (hemiarthroplasty and total hip arthroplasty) and anesthesia methods (PNB and SA) were also collected.

The independent variable in the present study was the anesthetic technique (PNB vs SA). The primary outcomes are all-cause mortality at 30 days, 90 days, and 1 year following hip arthroplasty. Causes of death were also recorded. Secondary outcomes included walking ability in the first postoperative year, LOS, cost of hospitalization, and major complications. All-cause mortality and walking ability in the first postoperative year were obtained by telephone follow-up. According to the functional independence measure scoring system, ${ }^{18}$ patients were classified as "independent walking" when they scored 5 points and over in the locomotion section. Patients who died within the first year after surgery were defined as walking ability impairment. Major complications included pulmonary infection, deep venous thrombosis in the lower limbs, pulmonary embolism, craniocerebral events, prosthetic joint infection, periprosthetic fracture, and dislocation.

\section{Treatment Protocol in Peri-Operative Period}

Patients were booked for urgent surgery as soon as the diagnosis was ascertained and major acute medical conditions were ruled out or reasonably addressed. Standard practice was to attempt to perform the surgical procedure within 48 hours after the diagnosis was confirmed. The details of PNB and SA procedures are presented below.

In the PNB group, three nerve blocks were conducted to ensure adequate anesthesia and analgesia during hip arthroplasties. Lumbar plexus, sciatic nerve, and first lumbar paravertebral nerve blocks were performed with a needle connected to an electrical nerve stimulation. The needle advanced until the contraction of femoral quadriceps muscle, and plantar or dorsal flexion of the foot were detected. The aforementioned nerves were further identified via handheld ultrasound, and the needle was inserted with an "in plane" approach. Subsequently, the ropivacaine with specified dosage and concentration was injected, namely $30 \mathrm{~mL}(0.3 \%), 20 \mathrm{~mL}(0.3 \%)$, and $8 \mathrm{~mL}$
$(0.25 \%)$ for lumbar plexus, sciatic nerve, and first lumbar paravertebral nerve blocks, retrospectively. Conscious sedation was achieved with dexmedetomidine, with concentrations of $1 \mathrm{ug} / \mathrm{kg}$ (intravenous drip within 10 minutes) at the beginning of anesthesia, and $4 \mathrm{ug} / \mathrm{kg} / \mathrm{h}$ during the surgery.

In the SA group, SA was performed with a $25 \mathrm{G}$ needle and 12.5-15 mg 0.5\% levobupivacaine. The L3-L4 or L4L5 intervertebral spaces were selected for spinal puncture. Local anesthetic ( $1 \mathrm{~mL} \mathrm{1 \%} \mathrm{lidocaine)} \mathrm{was} \mathrm{used} \mathrm{for} \mathrm{skin}$ infiltration. None of the patients received any sedation in the SA group.

Operations were performed through the standard posterolateral approach. Surgical procedures (hemiarthroplasty or total hip arthroplasty) were determined by surgeons under the consideration of patients' physical condition and acetabular morphology. If used, drainage tubes and catheters were removed within 24 hours following surgery. Antibiotics were initiated intraoperatively and maintained for 24-48 hours postoperatively for surgical infection prophylaxis. Low-molecular-weight heparin was administered for thromboprophylaxis. The standard postoperative active physiotherapy regimen was guided by an individual physiotherapist visiting once or twice a day. One week after surgery, partial weight-bearing was initiated. Full weight-bearing was allowed two weeks postoperation.

\section{Statistical Analysis}

Continuous data were expressed as mean \pm standard deviation or median with interquartile range. Categorical data were presented as percent (count). CCI was transformed into binary variable, and the median of CCI (4) was set as the cutoff. Baseline characteristics between the PNB and SA groups were summarized and compared using bivariate tests (Mann-Whitney U, $\chi^{2}$, and Fisher's exact tests). Patient demographics and baseline clinical data that could possibly affect postoperative morbidity and mortality were included, and a PSM analysis was performed with a ratio of 1:1. The rationale and methods of PSM to evaluate cause-and-effect relationships in retrospective studies have been well described. ${ }^{19}$ Briefly, the propensity score was calculated by logistic regression analysis using patient demographics and baseline clinical data to examine the association of anesthesia type with 30-day, 90-day, and one-year mortality. The tolerance error of PSM was 0.02. One-year survival analysis was performed using Kaplan-Meier analysis with Log rank test. Two-tailed tests were used, and differences were 
considered significant if $\mathrm{p}<0.05$. SPSS 20.0 statistical software (Chicago, IL, USA) was used for the analysis.

\section{Results}

\section{Demographic Data}

We identified 427 patients who underwent hip arthroplasty due to femoral neck fracture between January 1st, 2014 and December 31st, 2019. After screening, 316 patients were included in the final analysis; details are shown in the flowchart (Figure 1). Two hundred patients (63.3\%) received SA and 116 patients (36.7\%) received PNB. The unmatched groups differed in a number of baseline characteristics (Table 1). Patients who underwent SA were more likely to be younger, have a lower CCI score, have a spouse, smoke, to have been treated for total hip arthroplasty, and were less likely to receive anti-osteoporosis treatment before fracture.
Of the 316 patients, only $18.4 \%(n=58)$ underwent standardized anti-osteoporosis treatment before the fracture; details are shown in Table 2. One hundred and seventy-eight patients (89 per group) were selected after PSM. No significant difference in baseline characteristics was found between the two groups after matching (Table 1).

\section{Primary Outcomes}

As shown in Table 3, patients in the PNB group showed a significantly lower risk of death in 30 days $(2.2 \%$ vs $10.1 \%, \mathrm{P}=0.029)$ and 90 days $(3.4 \%$ vs $12.4 \%, \mathrm{P}=0.026)$ after hip arthroplasty, when compared to the SA group. Odds ratios of the SA group for 30- and 90-day mortality were 4.50 and 3.67, respectively. However, no significant difference was found regarding one-year mortality $(11.2 \%$ vs $16.9 \%$, $\mathrm{P}=0.281$ ). Kaplan-Meier analysis showed that one-year

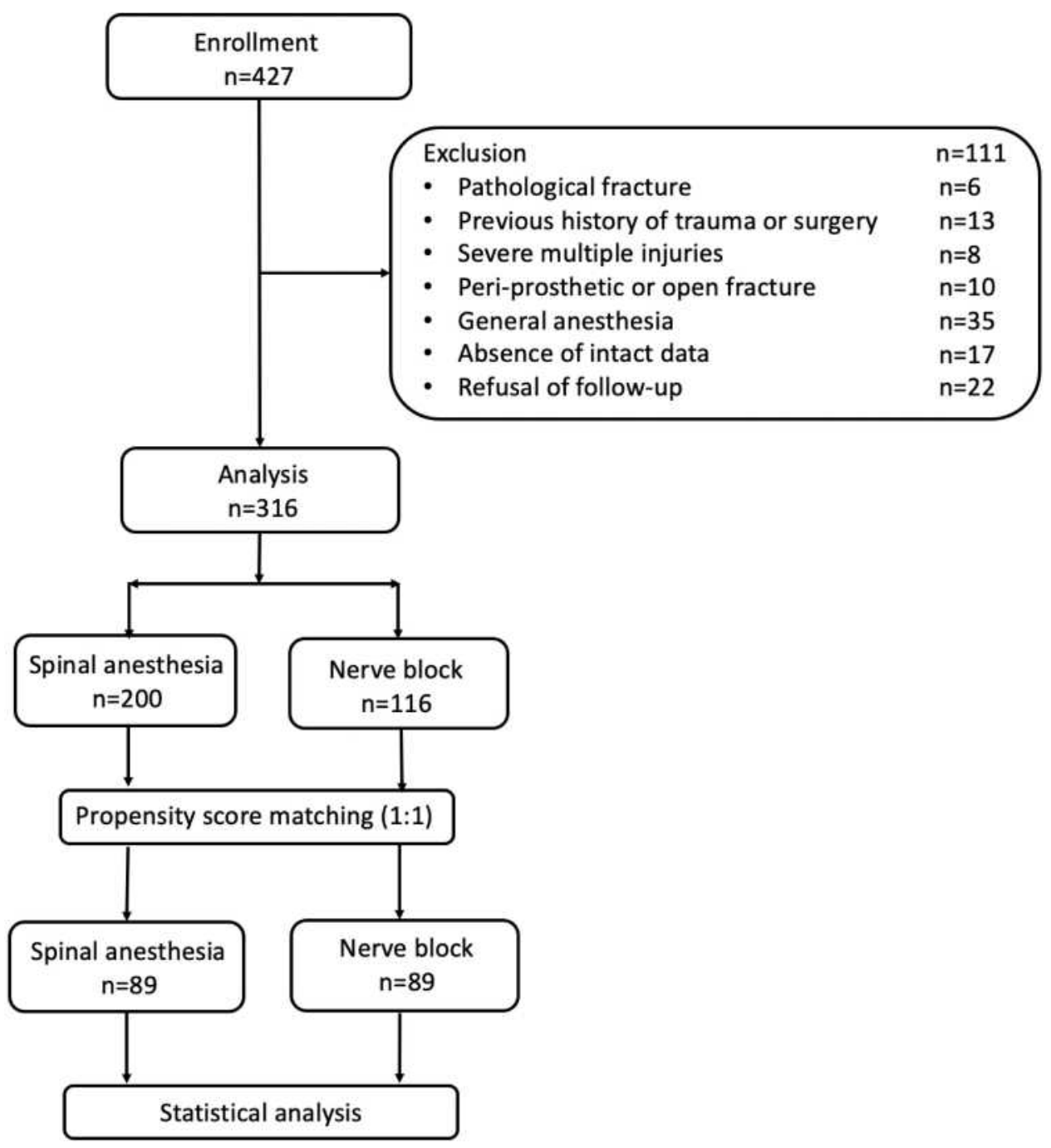

Figure I Flowchart of the present study. 
Table I Demographic Data

\begin{tabular}{|c|c|c|c|c|c|c|}
\hline \multirow[t]{2}{*}{ Items } & \multicolumn{3}{|c|}{ Before PSM } & \multicolumn{3}{|c|}{ After PSM } \\
\hline & $\begin{array}{l}\text { SA Group } \\
(n=200)\end{array}$ & $\begin{array}{l}\text { PNB Group } \\
(n=1 \mid 6)\end{array}$ & $P$ value & $\begin{array}{l}\text { SA Group } \\
(n=89)\end{array}$ & $\begin{array}{l}\text { PNB Group } \\
(n=89)\end{array}$ & $P$ value \\
\hline Age (years) & $76.5 \pm 1.1$ & $8 I . I \pm I .4$ & $<0.001$ & $77.3 \pm 1.7$ & $79.9 \pm 1.6$ & 0.105 \\
\hline Gender (male, \%) & $53(26.5 \%)$ & 21 (18.1\%) & 0.089 & 15 (16.9\%) & $18(20.2 \%)$ & 0.563 \\
\hline $\mathrm{CCl}(\leq 4, \%)$ & $132(66.0 \%)$ & 49 (42.2\%) & $<0.001$ & 37 (41.6\%) & $44(49.4 \%)$ & 0.292 \\
\hline Marriage (with spouse, \%) & I 48 (74.0\%) & $73(62.9 \%)$ & 0.039 & $62(69.7 \%)$ & $60(67.4 \%)$ & 0.747 \\
\hline Smoking history (no, \%) & 199 (99.5\%) & $112(96.6 \%)$ & 0.043 & 88 (98.9\%) & 88 (98.9\%) & 1.000 \\
\hline Resident (downtown, \%) & $164(82.0 \%)$ & 98 (84.5\%) & 0.572 & 70 (78.7\%) & $76(85.4 \%)$ & 0.242 \\
\hline Medical insurance (no, \%) & $83(41.5 \%)$ & $42(36.2 \%)$ & 0.354 & 36 (40.4\%) & 31 (34.8\%) & 0.439 \\
\hline Surgery (THA, \%) & $94(47.0 \%)$ & $20(17.2 \%)$ & $<0.001$ & 17 (19.1\%) & 17 (19.1\%) & 1.000 \\
\hline $\begin{array}{l}\text { Anti-osteoporosis treatment (no, } \\
\% \text { ) }\end{array}$ & $23(11.5 \%)$ & $35(30.2 \%)$ & $<0.001$ & $16(18.0 \%)$ & $17(19.1 \%)$ & 0.847 \\
\hline Fragility fractures (no, \%) & $188(94.0 \%)$ & $108(93.1 \%)$ & 0.752 & 85 (95.5\%) & $83(93.3 \%)$ & 0.515 \\
\hline $\begin{array}{l}\text { Time from injuries to surgery } \\
\text { (days) }\end{array}$ & $7(5,15)$ & $8(6,15)$ & 0.143 & $7(5,12)$ & $9(6,15)$ & 0.051 \\
\hline Baseline GLU (mmol/L) & $6.26 \pm 0.31$ & $6.67 \pm 0.46$ & 0.128 & $6.57 \pm 0.42$ & $6.30 \pm 0.50$ & 0.421 \\
\hline Baseline HGB $(\mathrm{g} / \mathrm{L})$ & $114.90 \pm 2.55$ & $116.96 \pm 3.20$ & 0.328 & $116.68 \pm 3.89$ & $116.52 \pm 3.72$ & 0.954 \\
\hline Baseline ALB (g/L) & $33.63 \pm 0.60$ & $33.35 \pm 0.77$ & 0.574 & $33.73 \pm 0.83$ & $33.26 \pm 0.93$ & 0.451 \\
\hline Baseline INR & $1.17 \pm 0.03$ & $1.24 \pm 0.27$ & 0.108 & $1.08 \pm 0.05$ & $1.08 \pm 0.03$ & 0.889 \\
\hline Baseline vital signs (normal, \%) & 145 (72.5\%) & $72(62.1 \%)$ & 0.054 & $58(65.2 \%)$ & $54(60.7 \%)$ & 0.535 \\
\hline Electrocardiograph (normal, \%) & $75(37.5 \%)$ & 43 (37.1\%) & 0.939 & $33(37.1 \%)$ & 31 (34.8\%) & 0.755 \\
\hline Chest radiography (normal, \%) & $43(21.5 \%)$ & $23(19.8 \%)$ & 0.724 & $18(20.2 \%)$ & 14 (I5.7\%) & 0.435 \\
\hline
\end{tabular}

Table 2 Details of Anti-Osteoporosis Treatment Before Fractures

\begin{tabular}{|l|c|c|}
\hline Items & $\mathbf{n}$ & $\%$ \\
\hline Standardized anti-osteoporosis therapy & 58 & $18.4 \%$ \\
Calcium + vitamin D + zoledronic acid & 22 & $7.0 \%$ \\
Calcium + vitamin D + alendronate & 17 & $5.4 \%$ \\
Calcium + vitamin D + teriparatide & 13 & $4.1 \%$ \\
Calcium + vitamin D + XianLingGuBao & 6 & $1.9 \%$ \\
\hline Non-standardized anti-osteoporosis therapy & 47 & $14.8 \%$ \\
Irregular medication usage & 33 & $10.4 \%$ \\
Calcium only & 14 & $4.4 \%$ \\
\hline Without anti-osteoporosis treatment & 211 & $66.8 \%$ \\
\hline
\end{tabular}

accumulated survival rate was higher in the PNB group without reaching a significant level (Figure 2). A total of 25 deaths were documented within the first year after surgery (15 in the
SA group and 10 in the PNB group); the causes of one-year mortality are summarized in Table 4 . Both matched groups had a similar incidence of cardio-cerebral vascular accidents, which also comprised the largest proportion.

\section{Secondary Outcomes}

Patients in the PNB group showed similar independent walking rate, LOS, and incidence of postoperative complications as compared with the SA group (Table 5). Pulmonary infection comprised the largest proportion of postoperative complications in both groups. The mean hospital cost for the SA group was significantly lower than that of the PNB group $(53,828.21$ CNY vs 59,278.83 CNY, $\mathrm{P}=0.024)$.

\section{Discussion}

In the present study, we found that there was a significant association between PNB and lower 30- and 90-day

Table 3 Comparison of 30-Day, 90-Day, and One-Year Mortality (n, \%)

\begin{tabular}{|l|c|c|c|c|}
\hline & SA Group (n=89) & PNB (n=89) & OR (95\% CI) & P value \\
\hline 30-day mortality & $9(10.1 \%)$ & $2(2.2 \%)$ & $4.50(1.00-20.24)$ & 0.029 \\
90-day mortality & II (12.4\%) & $3(3.4 \%)$ & $3.67(1.06-12.70)$ & 0.026 \\
One-year mortality & $15(16.9 \%)$ & $10(11.2 \%)$ & $1.50(0.71-3.16)$ & 0.281 \\
\hline
\end{tabular}




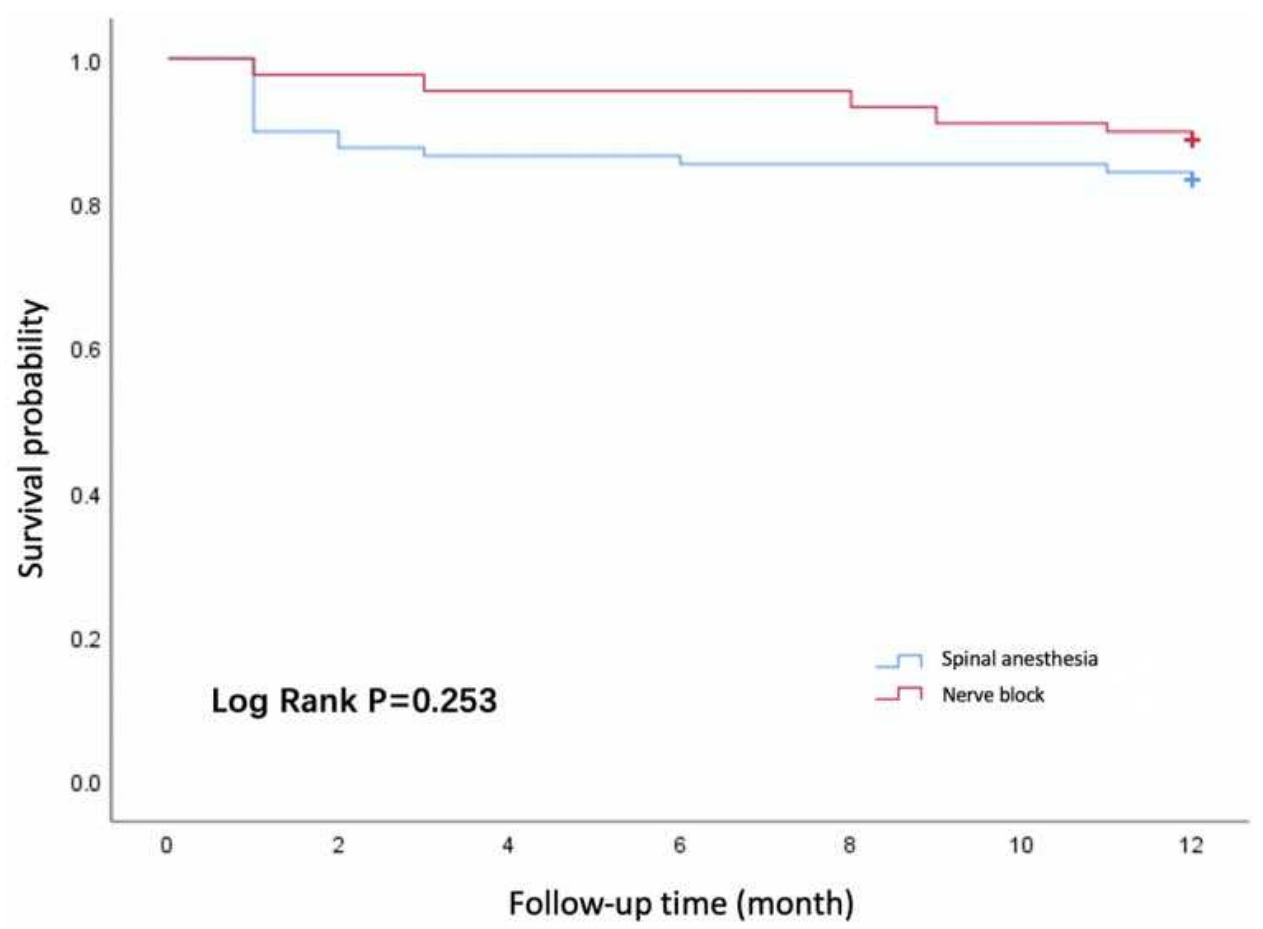

Figure 2 Cumulative postoperative mortality over time. The $Y$ axis represents mortality expressed as a percentage of all cases. The $X$ axis represents the number of postoperative months. The blue curve corresponds to patients who underwent spinal anesthesia, while the red curve corresponds to those who underwent peripheral nerve block.

mortality. It has been suggested that the advantage of PNB in postoperative mortality is related not only to its opioidsparing effect and decreased risk of opioid-related adverse event, but also lower risks of cognitive dysfunction, pneumonia, and hemodynamic instability due to short time of ambulation. $^{20}$ Only a few retrospective studies have focused on whether PNB can improve the postoperative outcomes of elderly patients with hip fractures, but their results remain conflicting. Patients with femur and hip fractures have shown benefits including morbidity and mortality advantages with PNB techniques. ${ }^{21}$ Karaca's

Table 4 Causes of One-Year Mortality

\begin{tabular}{|l|c|c|c|c|}
\hline \multirow{2}{*}{ Causes } & \multicolumn{2}{|c|}{$\begin{array}{c}\text { SA GRoup } \\
\text { (n=89) }\end{array}$} & \multicolumn{2}{c|}{$\begin{array}{c}\text { PNB Group } \\
(\mathbf{n = 8 9 )}\end{array}$} \\
\cline { 2 - 5 } & $\mathbf{n}$ & $\%$ & $\mathbf{n}$ & $\%$ \\
\hline Malignant tumor & 2 & 13.3 & 2 & 20.0 \\
Pulmonary infection & $\mathrm{I}$ & 6.7 & $\mathrm{I}$ & 10.0 \\
Kidney failure & 2 & 13.3 & 0 & 0.0 \\
Cardio-cerebral vascular accidents & 6 & 40.0 & 3 & 30.0 \\
Natural death & $\mathrm{I}$ & 6.7 & 2 & 20.0 \\
Pulmonary embolism & $\mathrm{I}$ & 6.7 & 0 & 0.0 \\
Undetermined & 2 & 13.3 & 2 & 20.0 \\
Total & 15 & 100 & 10 & 100 \\
\hline
\end{tabular}

study found that 30-day mortality was lower in the PNB group than the non-PNB group (19.1\% vs $8 \%$ ), in line with our findings. ${ }^{22}$ But other studies, conducted as secondary outcomes without adjusted analysis, found no significant difference in 30-day mortality between PNB and non-PNB groups. ${ }^{13,23}$ It is well known that one common limitation

Table 5 Comparison of the Secondary Outcomes in the Ist Postoperative Year

\begin{tabular}{|c|c|c|c|}
\hline Items & $\begin{array}{c}\text { SA Group } \\
(n=89)\end{array}$ & $\begin{array}{c}\text { PNB Group } \\
(n=89)\end{array}$ & $P$ value \\
\hline $\begin{array}{l}\text { Independent walking } \\
(\mathrm{n}, \%)\end{array}$ & 72 (80.9\%) & 63 (70.8\%) & 0.115 \\
\hline Complications (n, \%) & 7 (7.9\%) & $9(10.1 \%)$ & 0.600 \\
\hline $\begin{array}{l}\text { Pulmonary infection } \\
(\mathrm{n}, \%)\end{array}$ & 4 (57.1\%) & 4 (44.4\%) & \\
\hline DVT/PE (n, \%) & I (14.3\%) & $3(33.3 \%)$ & \\
\hline $\begin{array}{l}\text { Prosthesis } \\
\text { dislocation (n, \%) }\end{array}$ & $0(0)$ & $2(22.2 \%)$ & \\
\hline $\begin{array}{l}\text { Prosthetic joint } \\
\text { infection (n, \%) }\end{array}$ & $2(28.6 \%)$ & $0(0)$ & \\
\hline Hospital stays (days) & $12.3 \pm 1.3$ & $11.8 \pm 1.3$ & 0.569 \\
\hline $\begin{array}{l}\text { Hospitalization costs } \\
\text { (CNY) }\end{array}$ & $\begin{array}{l}53,828.21 \\
\pm 2552.48\end{array}$ & $\begin{array}{l}59,278.83 \\
\pm 4008.94\end{array}$ & 0.024 \\
\hline
\end{tabular}


of observational studies is selection bias, a consequence of lack of randomization, which results in higher-risk patients being more likely to undergo one of the interventions due to the theorized benefits. The retrospective studies in question enrolled patients with different types of hip fracture (femoral neck vs intertrochanteric fracture), surgery options (total hip arthroplasty, hemiarthroplasty, and internal fixation), and comorbidities between the PNB and nonPNB groups. Since mortality and other functional outcomes of elderly patients with hip fracture were affected by many patient-specific and treatment-related factors, ${ }^{24,25}$ we believed that selection bias might be a plausible explanation for the conflicting results between previous studies and our own.

PSM, used in the present study, is one of the main statistical approaches to minimizing selection bias. PSM offers certain advantages over more traditional regression methods to control for confounding by indication in observational studies. $^{26}$ By estimating the treatment effect through modeling the relationship between confounders and treatment assignment, the subgroups created after PSM were similar with respect to several meaningful covariates that are a priori known to affect the outcome of interest. ${ }^{26,27}$ Presumably, data after PSM better approximates what a prospective randomized data set would have shown. ${ }^{15}$ To minimize the selection bias in the present study, our PSM model included 18 independent variables that were either proven to influence or were likely to influence mortality. Most of the well-known preoperative indicators for postoperative mortality that were described in the previous studies, ${ }^{17}$ such as gender, age, CCI, residence, and preoperative ECG results, were also included. Furthermore, we enrolled qualified recent cases (2014-19) to avoid potential confounding from unmeasured and unknown effects of changes in processes of treatment and postoperative care over the study period. In these respects, our study likely has a higher standard of evidence than other retrospective studies. However, prospective randomized controlled studies are warranted for further investigation of this issue.

Although it was reported that better walking and stair climbing ability was found in the PNB group at 6 weeks after surgery, ${ }^{28}$ we found that the advantage of PNB on mortality and walking ability disappeared when it came to the first year after surgery. It has been reported that functional recovery after hip fracture may be largely complete in the first 6 months, extending up to 9 months for subjective recovery. ${ }^{29}$ Accordingly, we propose that this type of anesthesia has a limited effect on one-year mortality, as nearly all patients could fully or at least partially recover from the surgery and return to normal life at 6 months post-operation. We also found that cardio-cerebral vascular accidents accounted for a smaller proportion of causes of death in the PNB group, while lower risk of perioperative hemodynamic instability in the PNB group might be closely related. $^{7}$

The present study obtained different results from those conducted in North America, which found that receipt of PNB for hip fracture surgery is associated with decreased LOS and health system costs. ${ }^{23}$ We hypothesize that this discrepancy is attributable to differences in health care systems. Chinese patients typically return home after full recovery, rather than transfer to a rehabilitation center in a short period after surgery, which leads to higher cost and prolonged LOS.

We also noted that there was a relatively high mean waiting time from injury to surgery in the present study. In fact, all elderly hip fracture patients who were first admitted to our center underwent surgical interventions within $72 \mathrm{~h}$ after the diagnosis in the recent 4 years (2017-2021). However, some patients with severe comorbidities upon whom it was deemed unsafe to perform surgeries in local hospitals were transferred to our center. This would appear to be a reasonable explanation as the screening and transfer procedures in local hospitals are often timeconsuming (eg some cases might have a waiting time of over 100 days). Additionally, the practice of early surgery for hip fracture was widely adopted in our center after early 2017. Thus, some cases between 2014 and 2016 had a slightly longer waiting time, which might be another potential cause.

Hip fracture is one of the major complications of osteoporosis; standardized anti-osteoporotic therapy has been associated with decreased postoperative mortality for elderly patients with hip fracture. ${ }^{30}$ Consistent with an earlier study by Compston et al, ${ }^{31}$ we also found that only $18 \%$ of elderly hip fracture patients had standardized anti-osteoporosis treatment prior to injury. Orthopedic surgeons need to pay more attention to osteoporosis treatment to obtain better clinical outcomes and long-term prognosis for elderly patients with hip fractures.

Our study was subject to certain limitations. First, it is a single-center, retrospective study with a relatively small sample size. Although we performed PSM to minimize the potential influence of selection bias, we acknowledge that a larger sample size is strongly 
needed. Second, sole PNB for a hip fracture is a challenging procedure with multiple steps; as such, operator bias may be inevitable in the present study. Additionally, the PNB group had a lower 30-day mortality but a higher hospitalization cost in our study. We propose that a higher charge for PNB in our center might be a potential explanation. This hypothesis could not be validated as we did not obtain access to the itemized list of hospitalization costs, but only the total amount. Lastly, it was proven that dementia predicted one-year mortality for patients with hip fracture. ${ }^{32,33}$ However, we could not enroll this variable due to the lack of quantitative assessment of preoperative cognitive impairment in the original medical record.

\section{Conclusion}

We conducted a retrospective analysis with PSM design and found that PNB was related to lower 30- and 90-day mortality but higher hospitalization cost in elderly hip fracture patients after hip arthroplasty. However, PNB was not the factor that affected one-year mortality and walking ability, as well as major complications, and LOS. Orthopedic surgeons must pay greater attention to osteoporosis treatment to obtain better clinical outcomes and long-term prognosis for elderly patients with hip fractures.

\section{Abbreviations}

PNB, peripheral nerve block; SA, spinal anesthesia; PSM, propensity score matching; LOS, length of stay; ERAS, enhanced recovery after surgery; CCI, Charlson comorbidity index; ALB, serum albumin; INR, international normalized ratio.

\section{Data Sharing Statement}

The datasets used and/or analyzed during the current study are available from the corresponding authors on reasonable request.

\section{Ethics Approval}

The present study was conducted in accordance with the ethical principles of the Helsinki declaration and was approved by the institutional review board of Guangdong Provincial People's Hospital. Patient data in our study complied with relevant data protection and privacy regulations.

\section{Consent to Participate}

Signed informed consents for participation were unavailable due to the retrospective design, and the institutional review board of Guangdong Provincial People's Hospital has waived the informed consent procedure for the present study.

\section{Author Contributions}

All authors made a significant contribution to the work reported, whether that is in the conception, study design, execution, acquisition of data, analysis and interpretation, or in all these areas; took part in drafting, revising or critically reviewing the article; gave final approval for the version to be published; have agreed on the journal to which the article has been submitted; and agreed to be accountable for all aspects of the work.

\section{Funding}

This work was supported by Medical Scientific Research Foundation of Guangdong Province of China (A2021310), Project of Administration of Traditional Chinese Medicine of Guangdong Province of China (20211007), Natural Science Foundation of Guangdong Province (2021A1515011008), and the Program of Science and Technology of Guangzhou (201904010424).

\section{Disclosure}

The authors have no conflicts of interest.

\section{References}

1. Bhandari M, Swiontkowski M, Solomon CG. Management of acute hip fracture. $N$ Engl J Med. 2017;377(21):2053-2062. doi:10.1056/ NEJMcp1611090

2. Le Manach Y, Collins G, Bhandari M, et al. Outcomes after hip fracture surgery compared with elective total hip replacement. JAMA. 2015;314(11):1159-1166. doi:10.1001/jama.2015.10842

3. Pincus D, Ravi B, Wasserstein D, et al. Association between wait time and 30-day mortality in adults undergoing hip fracture surgery. JAMA. 2017;318(20):1994-2003. doi:10.1001/jama.2017.17606

4. Ahn EJ, Kim HJ, Kim KW, Choi HR, Kang H, Bang SR. Comparison of general anaesthesia and regional anaesthesia in terms of mortality and complications in elderly patients with hip fracture: a nationwide population-based study. BMJ Open. 2019;9(9):e029245. doi:10.1136/ bmjopen-2019-029245

5. Neuman MD, Rosenbaum PR, Ludwig JM, Zubizarreta JR, Silber JH. Anesthesia technique, mortality, and length of stay after hip fracture surgery. JAMA. 2014;311(24):2508-2517. doi:10.1001/jama.2014.6499

6. Rozenfeld M, Bodas M, Shani M, et al. National study: most elderly patients benefit from earlier hip fracture surgery despite co-morbidity. Injury. 2020;52(4):905-909. doi:10.1016/j.injury.2020.10.060

7. Hannon CP, Keating TC, Lange JK, Ricciardi BF, Waddell BS, Valle Della CJ. Anesthesia and analgesia practices in total joint arthroplasty: a survey of the American association of hip and knee surgeons membership. $J$ Arthroplasty. 2019;34(12):2872-2877.e2872. doi:10.1016/j.arth.2019.06.055 
8. Guay J, Parker MJ, Griffiths R, Kopp S. Peripheral nerve blocks for hip fractures. Cochrane Database Syst Rev. 2017;5(5):CD001159. doi:10.1002/14651858.CD001159.pub2

9. Unneby A, Svensson O, Gustafson Y, Olofsson B. Femoral nerve block in a representative sample of elderly people with hip fracture: a randomised controlled trial. Injury. 2017;48(7):1542-1549. doi:10.1016/j.injury.2017.04.043

10. Skjold C, Møller AM, Wildgaard K. Pre-operative femoral nerve block for hip fracture-A systematic review with meta-analysis. Acta Anaesthesiol Scand. 2020;64(1):23-33. doi:10.1111/aas.13491

11. Howard CB, Mackie IG, Fairclough J, Austin TR. Forum. Femoral neck surgery using a local anaesthetic technique. Anaesthesia 1983;38(10):993-994. doi:10.1111/j.1365-2044.1983.tb12032.x

12. Ho AMH, Karmakar MK. Combined paravertebral lumbar plexus and parasacral sciatic nerve block for reduction of hip fracture in a patient with severe aortic stenosis. Can J Anaesth. 2002;49(9):946-950. doi:10.1007/BF03016880

13. Jin J, Wang G, Gong M, Zhang H, Liu J. Retrospective comparison of the effects of epidural anesthesia versus peripheral nerve block on postoperative outcomes in elderly Chinese patients with femoral neck fractures. Clin Interv Aging. 2015;10:1223-1231. doi:10.2147/CIA. S87020

14. Johnston DF, Stafford M, McKinney M, Deyermond R, Dane K. Peripheral nerve blocks with sedation using propofol and alfentanil target-controlled infusion for hip fracture surgery: a review of 6 years in use. J Clin Anesth. 2016;29:33-39. doi:10.1016/j. jclinane.2015.10.012

15. Reiffel JA. Propensity score matching: the "Devil is in the Details" where more may be hidden than you know. Am J Med. 2020;133 (2):178-181. doi:10.1016/j.amjmed.2019.08.055

16. Nakajo K, Abe S, Oda I, et al. Impact of the Charlson comorbidity index on the treatment strategy and survival in elderly patients after non-curative endoscopic submucosal dissection for esophageal squamous cell carcinoma: a multicenter retrospective study. J Gastroenterol. 2019;54(10):871-880. doi:10.1007/s00535-01901583-9

17. Zanker J, Duque G. Rapid geriatric assessment of hip fracture. Clin Geriatr Med. 2017;33(3):369-382. doi:10.1016/j.cger.2017.03.003

18. D'Andrea A, Le Peillet D, Fassier T, et al. Functional independence measure score is associated with mortality in critically ill elderly patients admitted to an intermediate care unit. BMC Geriatr. 2020;20(1):334-338. doi:10.1186/s12877-020-01729-y

19. Malhas L, Perlas A, Tierney S, Chan VWS, Beattie S. The effect of anesthetic technique on mortality and major morbidity after hip fracture surgery: a retrospective, propensity-score matched-pairs cohort study. Reg Anesth Pain Med. 2019;44(9):847-853. doi:10.1136/rapm-2019-100417

20. Gottlieb M, Long B. Peripheral nerve block for hip fracture. Acad Emerg Med. 2021. doi:10.1111/acem.14239

21. Gadsden J, Warlick A. Regional anesthesia for the trauma patient: improving patient outcomes. Local Reg Anesth. 2015;8:45-55. doi:10.2147/LRA.S55322

Clinical Interventions in Aging

\section{Publish your work in this journal}

Clinical Interventions in Aging is an international, peer-reviewed journal focusing on evidence-based reports on the value or lack thereof of treatments intended to prevent or delay the onset of maladaptive correlates of aging in human beings. This journal is indexed on PubMed Central, MedLine, CAS, Scopus and the Elsevier
22. Karaca S, Ayhan E, Kesmezacar H, Uysal O. Hip fracture mortality: is it affected by anesthesia techniques? Anesthesiol Res Pract. 2012;2012:708754. doi: $10.1155 / 2012 / 708754$

23. Hamilton GM, Lalu MM, Ramlogan R, et al. A population-based comparative effectiveness study of peripheral nerve blocks for hip fracture surgery. Anesthesiology. 2019;131(5):1025-1035. doi:10.1097/ALN.0000000000002947

24. Malafarina V, Reginster J-Y, Cabrerizo S, et al. Nutritional status and nutritional treatment are related to outcomes and mortality in older adults with hip fracture. Nutrients. 2018;10(5):555. doi:10.3390/ nu10050555

25. Huette P, Abou-Arab O, Djebara A-E, et al. Risk factors and mortality of patients undergoing hip fracture surgery: a one-year follow-up study. Sci Rep. 2020;10(1):9607-9608. doi:10.1038/s41598-02066614-5

26. Benedetto U, Head SJ, Angelini GD, Blackstone EH. Statistical primer: propensity score matching and its alternatives. Eur J Cardiothorac Surg. 2018;53(6):1112-1117. doi:10.1093/ejcts/ ezy 167

27. Yang JY, Webster-Clark M, Lund JL, Sandler RS, Dellon ES, Stürmer T. Propensity score methods to control for confounding in observational cohort studies: a statistical primer and application to endoscopy research. Gastrointest Endosc. 2019;90(3):360-369. doi:10.1016/j.gie.2019.04.236

28. Morrison RS, Dickman E, Hwang U, et al. Regional nerve blocks improve pain and functional outcomes in hip fracture: a randomized controlled Trial. J Am Geriatr Soc. 2016;64(12):2433-2439. doi:10.1111/jgs.14386

29. Fischer K, Trombik M, Freystätter G, Egli A, Theiler R, BischoffFerrari HA. Timeline of functional recovery after hip fracture in seniors aged 65 and older: a prospective observational analysis. Osteoporos Int. 2019;30(7):1371-1381. doi:10.1007/s00198-01904944-5

30. Wang P-W, Li Y-Z, Zhuang H-F, et al. Anti-osteoporosis medications associated with decreased mortality after hip fracture. Orthop Surg. 2019;11(5):777-783. doi:10.1111/os.12517

31. Compston J, Cooper A, Cooper C, et al. UK clinical guideline for the prevention and treatment of osteoporosis. Arch Osteoporos. 2017;12 (1):43. doi:10.1007/s11657-017-0324-5

32. Chiu H-C, Chen C-M, Su T-Y, et al. Dementia predicted one-year mortality for patients with first hip fracture: a population-based study. Bone Joint J. 2018;100-B(9):1220-1226. doi:10.1302/0301620X.100B9.BJJ-2017-1342.R1

33. Dutkiewicz R, Zetterberg H, Andreasson U, Blennow K, Nellgård $B$. Dementia and CSF-biomarkers for Alzheimer's disease predict mortality after acute hip fracture. Acta Anaesthesiol Scand. 2020;64 (1):93-103. doi:10.1111/aas.13472
Bibliographic databases. The manuscript management system is completely online and includes a very quick and fair peer-review system, which is all easy to use. Visit http://www.dovepress.com/ testimonials.php to read real quotes from published authors. 